\title{
The Dominant Cognitive Processing Route in Literary and Subtitle Translation: A Case Study of Wolf Totem
}

\author{
Xiaodong Liu*, Xiangyan Zhou \\ School of Foreign Studies, Hunan University of Humanities, Science and Technology, Loudi, China \\ Email address: \\ 18063868429@163.com (Xiaodong Liu),934590502@qq.com (Xiangyan Zhou) \\ ${ }^{*}$ Corresponding author \\ To cite this article: \\ Xiaodong Liu, Xiangyan Zhou. The Dominant Cognitive Processing Route in Literary and Subtitle Translation: A Case Study of Wolf Totem. \\ International Journal of Applied Linguistics and Translation. Vol. 6, No. 3, 2020, pp. 96-102. doi: 10.11648/j.ijalt.20200603.17
}

Received: September 8, 2020; Accepted: September 19, 2020; Published: September 30, 2020

\begin{abstract}
This article draws on a neurofunctional theory of bilingual processing and its application to translation to investigate the dominant cognitive processing route in the literary and subtitle translation and also differences of the dominant patterns between the two translation modes, specifically those involving Chinese-English language pairs. This general investigation has two specific aims: 1) to extract the translation strategy patterns at the textual level and 2) to determine the dominant processing route at the cognitive level by linking the strategies to processing routes. Building on naturalistic translational data, a bilingual corpus was self-built; the linguistic features of the translated products were analyzed. The current study analyzes the renderings of Chinese culture-specific items into English. The results suggest that the dominant processing pattern can be identified in both the literary and subtitle translation. Moreover, based on the empirical evidence, it is found that the meaning-based processing route dominates both literary and subtitle translation. This article concludes that the contextual-intentional-conceptual system handles culture-specific concepts through conceptual mediation, which is required in the translation process due to contextual and pragmatic factors. The issue of cognitive processing routes that may take place in the translator's brain is of significance to translation process research specifically and translation studies in general.
\end{abstract}

Keywords: Culture-Specific Items, Wolf Totem, Meaning-Based Processing Route, Form-Based Processing Route

\section{Introduction}

The purpose of this article is to investigate the dominant processing pattern in literary and subtitle translation, specifically those involving Chinese-English language pairs. This investigation is conducted through the case analysis of rendering Chinese culture-specific items (CSIs) in Lang Tuteng (Wolf Totem) into their English counterparts. During the translation process, as Garcia states, there must be a transfer from the source language (SL) to the target language (TL) that occurs in the translators' brain [12]. This transfer has triggered the interest of translation studies researchers regarding the properties of translation processes as the goal of inquiry, such as $[2,11,14,16,22]$. Among all the questions raised regarding this topic, a pertinent question is how the SL is transferred into the TL during the translation process, i.e., the cognitive processing routes.

To date, there has been a surge of studies on the processing routes for SL-TL transfers, which have identified two kinds of mental procedures that may take place in translators' brains during translation tasks: form-based processing routes and meaning-based processing routes $[6-8,16,19,20]$. The form-based route refers specifically to the direct transfer from a source language to a target language based on lexical, phonological, and syntactical forms. However, the meaning-based route involves the transposition of meaning carried in the source text to that in the target text, which requires conceptual mediation in the translator's brain. In the literature, a difficult question remains unclear: are form-based or meaning-based processing routes the dominant route in literary translation and subtitle translation?

Drawing on the differences between literary translation and subtitle translation, we assume that the dominant processing pattern in these two situations is different. This paper tries to investigate whether the claim mentioned above can be empirically evaluated in both literary and subtitle situations. 
In the following, we seek to find the dominant processing patterns by looking at translations of Wolf Totem, which has been made publicly available in the form of both a published novel and an adapted movie.

\section{Literature Review: Cognitive Processing Routes in Translation Studies}

The past four decades have produced fruitful results regarding the processing routes for bilingual transfers in translators' brains as related to a hotly debated issue, i.e., which route dominates translation, the form-based processing route or the meaning-based processing route? Some scholars argue that the form-based processing route dominates translation, while others argue the opposite view. One particular group of researchers who have attempted to address this issue are those from translation studies, such as $[3,4,7,8$, $14,18,22]$.

Among the literature, there are several studies showing that meaning-based processing routes are frequently used during translation processes. For instance, Barik suggested that less proficient translators produce both less literal and more conceptually accurate translations, which indicates a tendency toward the conceptual mediation route [3]. This suggestion is echoed by the following two additional studies. $\mathrm{He}$ investigated translating patterns, i.e., the dominant translating strategy, of culturally indigenous concepts and found that historic idioms and conventional metaphors are often translated through meaning-based routes [14]. Ge also investigated the translation of alien source concepts in the humorous Chinese literary work Fortress Besieged by Qian Zhongshu and observed the following translating patterns of alien source concepts: paraphrasing $>$ transcoding $>$ substitution $>$ deletion $^{1}$ [13].

However, the findings of other studies indicate that professional interpreters tend to adopt form-based processing routes rather than meaning-based routes. For example, Dam examined one Spanish text (ST) and five consecutive interpretations (TTs) [7], as well as two Spanish STs and five simultaneous interpretations of each in Danish [8]. The author found that the form-based route was employed more in both the CI and the SI. These results corroborate part of the findings of Liu, Li \& He [18], who also investigated the processing patterns in professional Chinese-English consecutive interpretations. In terms of written translations, Chou, Lei, Li \& He [4] explored the translation patterns for culture-specific items extracted from a self-built bilingual parallel corpus totaling approximately one million words, including an English novel, The Joy Luck Club by Amy Tan, and its four Chinese translations. Transcoding dominates the use of translation strategies for CSI-related units in four individual translations. Due to the accumulated empirical evidence, the form-based route is

1 ">" here simply means "is used more than". argued to be the default translation procedure among professional translators [22]. It seems that the dominant route heavily depends on the mode of translation, the text typology, and other factors.

What we can gain from the abovementioned studies is of much significance. First, previous research has involved different modes of translation, including written translation and interpreting. Second, the conflicting findings are controversial, implying that the issue of processing routes needs to be empirically investigated further. Third, although other evidence concerning this controversial issue in Chinese-English translation has also been collected in recent years, such as $[4,13,17,23]$, there are few systematic studies comparing literary and subtitle translation from Chinese into English (CE) regarding the dominant processing patterns. Therefore, the current investigation aims to further investigate this issue by expanding the research into the area of subtitles and exploring the tendencies of translations involving Chinese and English by adopting a corpus-assisted approach.

\section{Theoretical Underpinnings}

This section has three subsections. First, we introduce the cognitive systems of the human brain underlying natural language processing in a brief manner. Next, we present the theoretical concepts of translation under the framework of language processing in general and bilingual processing specifically. Finally, we establish the theoretical problem that will be explored empirically in this study.

\subsection{Cognitive System of the Human Brain}

In the human brain, there is a cognitive system, without which language processing is not possible. There assumed to be four components in the cognitive system that work to complete such language processing tasks as translating and interpreting, including the articulatory-perceptual system, the memory system, the language faculty, and the contextual-intentional-conceptual system (also referred to as the thought system) $[5,16,21]$.

The articulatory-perceptual system contains human organs such as the eyes, ears, hands, mouth, etc. In written translation, the translator has to receive the ST through their eyes and produce the TT through hands-based writing, while in interpreting, the interpreter must listen to the source speaker so that the input can be received by the ears, and then the interpreter delivers the target output through their mouth. The memory system is made up of long-term memory and short-term memory. In the long-term memory system, fixed expressions are stored, which can no longer be segmented.

The language faculty is of significance to language processing, which applies grammatical rules to generate phrases and sentences [16]. For translators and interpreters, the language faculty is bilingually represented and stored in their brains. The functions of language faculty are described as follows.

"The Language Faculty does two things: being connected to 
the thought systems and sensor-motors on the one hand, and generating structured expressions on the other. For those tasks, it has four components: Lexicon, Syntax, the Phonetic Interface (to sensor-motors) and the Logical Interface (to the thought systems)". [16]

The contextual-intentional-conceptual system stores concepts, intentions, etc. It initiates communications between different parties.

\subsection{Bilingual Processing and Translation}

For neurolinguists and cognitive psychologists, the properties of translation consist of bilingual processing. Thus, one of the fundamental issues to be examined is how the translator deals with the bilingual data when, for example, the input is delivered in one language (L1/L2), and the output is delivered in the other (L2/L1). According to previously presented theoretical proposals $[9,10,16,19,20]$, there seem to be two cognitive processing routes for translation and interpreting, namely, the meaning-based and form-based processing routes.

Theoretically speaking, for the meaning-based processing routes, the received source input (either by eyes or ears) is parsed in the language faculty and interpreted in the thought systems. The decoded source message is then conceptually mediated into the target message, after which it is encoded in the target language through the language faculty and verbalized by the articulatory-perceptual systems (the speech organs or hands, simply) [16]. The meaning-based route initially processes language bottom-up and then does so top-down, i.e., in a vertical manner. Hence, the route's other monikers, "vertical translation" [10] and "the conceptually mediated route" $[19,20]$.

In addition to meaning-based processing routes, professional translators or interpreters also engage in form-based processing, aka "horizontal translation" $[9,10]$ and "direct transcoding" $[19,20]$. When the meaning-based processing route is engaged, information is processed from the L1 into concepts and then into the L2, or vice versa. When the form-based route is engaged, the L1-L2 or L2-L1 system works to complete the translating/interpreting task more automatically without going to the thought system for conceptual mediation. There are lexical links in the lexicon, phonological links in the phonetic interface, and syntactic links in the grammar-parser [16]. The processes are economic and thus perceived as "shortcuts" and "translation-specific routes" $[12,16]$.

\subsection{Revisiting Cognitive Processing Routes in Translation}

From the perspective of bilingual processing, it is argued that 1) the form-based processing route does not involve the contextual-intentional-conceptual system, and it may be specific to professional translators. In addition, 2) for meaning-based processing routes, the translator or interpreter has to fully comprehend the source text; otherwise, the meanings carried in the source text cannot be extracted. Simply saying, these two cognitive processing routes produce different translations, which can be used to identify the performances of translators. Liu, Li \& He has shown that the interplay of these two routes is affected by various factors [18]. However, the study only focuses on professional consecutive interpretations. In the current paper, we attempt to address the issue of how the two processing routes play out in literary and subtitle situations, as presented below.

\section{Methodological Framework}

In this section, the source and the target material for the current research are presented. Then, a corpus-assisted approach to process research is introduced. Finally, the hypotheses are given.

\subsection{Materials}

The data used in this study are from the novel Wolf Totem. The data specifically include 1) the English translation of Wolf Totem by the American sinologist Howard Goldblatt (see Appendix I for details) and 2) the subtitle translations from the movie "Wolf Totem" filmed by French director Jean Jacques Annaud.

This work is targeted as our source of data for three reasons: 1) the book is enriched by both Han Chinese and Mongolian culture and is representative of that field of published books; 2) it has been absorbed in a range of readership across languages; i.e., the English version of "Lang Tu Teng" has been sold in over 110 countries; and 3 ) the novel was adapted into a movie, which provides a source of subtitle translations. Therefore, this study can compare translation patterns between literary and subtitle translation.

\subsection{Corpus-Assisted Approach to Processing Routes Research}

To investigate the processing patterns in translation, a bilingual corpus was self-built (see Appendix II), and the linguistic features of the translated products were analyzed. To be more specific, culture-specific items (CSIs), i.e., those items alien to the target system (system refers to the language and culture), were selected as a way to determine how the bilingual transfer is done during the act of translating. In particular, a bilingual parallel corpus is used to infer translators' or interpreters' underlying processing routes via the extraction of the rendering patterns of the CSIs, thus called a corpus-assisted approach to process route research, which has already been applied in some of the previous studies $[4,14$, 15].

Indeed, there are several theoretical questions related to this approach. The first and foremost question is why CSIs are selected for researching the processing routes. The next question is how the translation strategies for CSI translation are descriptively defined and classified.

CSIs are defined as "textually actualized items whose functions and connotations in a source text involve a translation problem in their transference to a target text, whenever this problem is a product of the nonexistence of 
the referred item or of its different intertextual status in the cultural system of the readers of the target text" [1]. They are unique to the source system and thus alien to the target system. Translating CSIs often poses conceptual barriers for the translator during the translation process [15]. For example, the Chinese sentence “我早上八点起床” can be easily translated into English "I get up at eight o'clock in the morning" while such idiomatic phrases as “马到成功” ("Someone will achieve success as the horse arrives") may be challenging. Because of the difficulties, translators are likely to leave processing traces in the translations. The CSIs examined in this study include proper names, metaphors, idiomatic expressions, and classic quotations. To obtain the translation strategy pattern at the textual level, the abovementioned issue of how to describe translation strategies needs to be addressed. The translation strategies and their definitions are summarized in Table 1.

Table 1. Translating Strategies.

\begin{tabular}{|c|c|}
\hline Paraphrasing & To translate by meaning \\
\hline Omission & No translation \\
\hline Transcoding & $\begin{array}{l}\text { To translate by structure (phonological, } \\
\text { morphological, or syntactic) }\end{array}$ \\
\hline
\end{tabular}

For the dominant processing patterns, a link is established between the translation strategies and the processing routes, as seen in Table 2.

Table 2. Cognitive Processing Routes and Their Corresponding Manifestations as Translation Strategies [17, 18].

\begin{tabular}{ll}
\hline Cognitive & Descriptive \\
\hline Meaning-based processing route & Paraphrasing \\
Form-based processing routes & Omission \\
\hline
\end{tabular}

\subsection{Hypotheses}

According to the literature review presented in Section 2 and the theoretical proposals in Section 3, two major working hypotheses are formulated and tested regarding the literary and subtitle translation. The first hypothesis is as follows:

(1) In pure theoretical terms, there are two processing routes in translation, meaning-based and form-based. The dominant processing pattern can be observed in the data involving subtitle translation and literary translation.

It is known that the differences between subtitle translation and literary translation are obvious. The former is restricted to time and space, while in the latter situation, the translator has enough time to complete the task and can do so without space limits. Thus, the second hypothesis is as follows:

(2) The patterns of processing routes between literary translation and subtitle translation are different due to the abovementioned differences.

In summary, we compare the literary and subtitle translation in terms of the processing routes for the SL-TL transfer in the current study. The transfer could be based on either form or meaning. The two encoding mechanisms are affected by many variables, such as different modalities. The results are presented in the next section.

\section{Results and Discussion}

As presented in the previous section, there are two cognitive processing routes for bilingual transfers in the translation processes, i.e., form-based and meaning-based routes. It is found in the current study that the dominant route in literary and subtitle translation is the same, i.e., meaning-based processing route. Next, the results are described in three sub-sections: the literary translation, the subtitle translation, and the comparison between these two.

\subsection{Processing Pattern in the Literary Translation}

In this section, we investigate the translation processing pattern in the literary translation. A total of 380 instances of CSIs were selected from the self-built sub-corpus of the novel. A table concerning the translation pattern in the Chinese-English literary translation is visualized below.

Table 3. A Translating Pattern for CSIs in Wolf Totem: Chinese-English Literary Translation.

\begin{tabular}{lll}
\hline Cognitive Processing Routes & Frequency & Percentage (\%) \\
\hline Meaning-based Processing Route & 188 & $49.48 \%$ \\
Form-based Processing Route & 157 & $41.31 \%$ \\
Omission & 35 & $9.21 \%$ \\
Total & 380 & $100 \%$ \\
\hline
\end{tabular}

As seen in Table 3, the processing pattern in the literary translation is meaning-based route $(49.48 \%)>$ form-based processing route $(41.31 \%)>$ omission $(9.21 \%)$. This effect seems to indicate that the meaning-based route is dominant in the professional Chinese-English literary translation. The pattern in the current subsection agrees with some of the findings of previous studies [3, 13, 14]. A question arises from the pattern: why is the meaning-based processing route employed more than the form-based processing route in the literary translation? What does this result reveal about the translation processes? Let us take a look at a few examples first.

(1) Rendered by the meaning-based processing route: ST: 距他不到 40 米的雪坡上, 在晚霞的天光下, 竟 然出现了一大群金毛灿灿、杀气腾腾的蒙古狼。

TT: There on a snow-covered slope not less than fifty yards away was a pack of golden-hued, murderous-looking Mongolian wolves.

(2) Rendered by the form-based processing route:

ST: 烧草原, 犯天条, 熏黑了腾格里的脸, 腾格里 还会给人好脸色看吗?

TT: “A grassfire violates heavenly laws," Bilgee said. "It blackens the face of Tengger, and you know what that will mean for us.

(3) Rendered by omission:

ST:...狗屁不通, 狗娘养的, 狗仗人势, 狗急跳墙, 鸡狗升天.. 
TT: No translation.

As we see here, in Example (1), the Chinese idiomatic expressions “金毛灿灿” and “杀气腾腾” were translated into "golden-hued" and "murderous-looking", respectively, through meaning-based routes. The source form has been lost, but the translations are familiar to the target readers. In Example (2), the translator employed a form-based processing route to transfer the Chinese phrase “犯天条” into "violates heavenly laws" and the proper name “腾格里” into “Tengger", which indeed keeps the original flavor of the source. However, the translations pose difficulties to the readers (e.g., what are heavenly laws and who is Tengger?). In addition to the previous two routes that produce translations, omission is also used to translate such Chinese idiomatic expressions as “狗屁不通” and “狗娘养的” in Example (3). Different processing routes produce different translations exerting various effects on the target readers. Let us go back to the question posed previously: why is the meaning-based processing route used over the form-based processing route in the examined literary translation?

When we try to answer this question, we may first focus on the nature of the meaning-based processing route. As we already know, meaning-based translations come from paraphrasing, and thus, the form is lost. To transfer the information embodied in the ST, much conceptual mediation is done in the thought system so that the message can be made compatible with the conceptualization of the target environment. Simply speaking, the target readers can understand the mediated message. It is acknowledged that the ultimate purpose of the English translation of Wolf Totem is for the sake of the native English speaker, and thus communication between English-speaking people and the novel can be successfully achieved. Therefore, the translator tends to adopt a more meaning-based processing route, which produces paraphrased texts that are easier to comprehend.

\subsection{Processing Pattern in Subtitle Translation}

For the subtitle translation situation, 87 CSIs were extracted from the movie subtitles in Wolf Totem. The figure concerning the translation patterns in the Chinese-English subtitle translation is shown in Table 4.

Table 4. A Translating Pattern for CSIs in Wolf Totem: Chinese-English Subtitle translation.

\begin{tabular}{lll}
\hline Cognitive Processing Routes & Frequency & Percentage (\%) \\
\hline Meaning-based Processing Route & 49 & $56.32 \%$ \\
Form-based Processing Route & 37 & $42.53 \%$ \\
Omission & 1 & $1.15 \%$ \\
Total & 87 & $100 \%$ \\
\hline
\end{tabular}

As seen in Table 4, the processing pattern in subtitle translation is meaning-based route $(56.32 \%)>$ form-based processing route $(42.53 \%)>$ omission $(1.15 \%)$. The processing pattern indicates that the meaning-based route is dominant in the professional Chinese-English subtitle translation. The pattern observed in the current subtitle situation agrees with some of the findings of previous studies as well $[3,13,14]$. This pattern poses the same question as that above: why is the meaning-based processing route employed more than the form-based processing route in subtitle translation? What does this result reveal about the translation processes? Before we try to understand the translation processes in subtitle translation, a few examples ${ }^{2}$ are given below.

(4) Rendered by the meaning-based processing route: $\mathrm{ST}$ :成为第一批下乡的知青

TT: I was among the very first to go

(5) Rendered by the form-based processing route: $\mathrm{ST}$ :这是上乌拉盖的车吗

TT: Is this the bus to Wulagai

(6) Rendered by omission: $\mathrm{ST}$ :城里的学生被下放到农村去插队

TT: City students were sent to the countryside (No translation for this CSI)

In Example (4), the Chinese culture-specific expression “下 乡的知青” (“the knowledgeable youth who go to the villages for work") was paraphrased into "among the very first to go". The culture-specific concept carried in the Chinese expression is conceptually mediated and thus lost. In Example (5), the proper name “乌拉盖” was simply transliterated into "Wulagai" through its phonological form. In addition to the previous two routes, omission is also used to translate such Chinese culturally indigenous expressions as “插队”, which are often used in the period of the Chinese cultural revolution. Compared to the literary translation examples, the subtitle translation examples are short and less difficult, as evidenced by fewer CSIs. Again, let us go back to the same question posed above: why do meaning-based processing routes prevail over form-based processing routes in subtitle translation?

As previously presented, the meaning-based processing route may help the translator maintain the message through mediation. For subtitle translation, two restrictions must be imposed on the translator, namely, the time and space limits. The source and target are often placed within a single line in a respective manner. It would be easy enough to catch the audience's eyes. Moreover, the audience cannot go back and revisit the source. From the audience's perspective, the translator tends to paraphrase the culture-specific concepts alien to them. Due to these factors, the translations need to be very clear and easily understood.

\subsection{A Comparative Analysis of Literary and Subtitle Translation}

This section presents a comparison of the processing patterns between literary translation and subtitle translation. As we know, for literary translation, the source is always there, while subtitles are transient. In subtitle translation, both the time and space for the translation are strictly restricted. Due to such differences, the question is as follows: are there any differences in the processing patterns of translating the two different types of sources? The patterns of comparison are presented below.

\footnotetext{
2 In the examples there are no punctuations since the data has been extracted from the subtitles, which are presented in the movie utterances by utterances.
} 


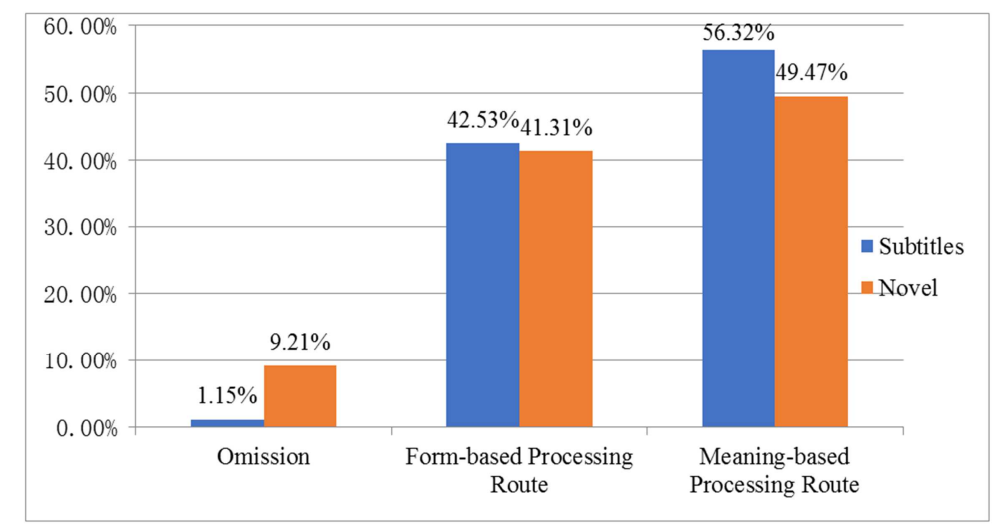

Figure 1. A Translating Pattern for CSIs in Wolf Totem: Chinese-English Literary and Subtitle Translation.

As Figure 1 shows, the dominant processing pattern is the meaning-based route in both subtitle translation $(56.32 \%$ vs $42.53 \%)$ and literary translation $(49.47 \%$ vs $41.31 \%)$. It was predicted that the processing patterns would be different, so the concern here is why these two different sources share the same processing pattern.

This can be explained in the following ways. First, regardless of the situation, i.e., either subtitle or literary translation, the ultimate purpose is to make communication possible between novels and English readers (in literary situations) and between films and English-speaking audiences (in subtitle situations). Therefore, the translator assumes the delivery of the message carried in the source as a priority and leaves the form aside when possible. From this angle, the modality differences are compromised. Second, the processing differences in these two situations may not be reflected in the textual patterns and may only be found through the application of other cognitive measures. Finally, the amount of translation experience and the translator's training may modulate the operations of the processing routes. As we have discussed in the previous sections, translators of lower proficiency often rely on meaning-based routes [3].

However, one point is worth noting, i.e., why are omissions in subtitle translations less frequent than those in literary translations? This may be attributable to contextual factors. In a literary translation, if some concepts that are alien to the target readers are deleted, it may not affect the readers' understanding of the message because the overall context remains. However, for subtitle translation, the subtitles appear for only a few seconds. In such a short period of time, the message cannot be fully understood if too many omissions take place without the context being available.

\section{Conclusions}

In the translation process, there are two cognitive processing routes for bilingual transfer: the form-based processing route and the meaning-based processing route $[8,10,16]$. The controversial issue of dominance regarding the workings of these routes has not been well addressed in the field of subtitle translation, although empirical evidence has been found for written translation $[4,14]$, consecutive interpreting $[7,17]$ and simultaneous interpreting [8]. In previous studies, the dominant pattern of cognitive processing routes has been shown to be related with many variables, such as the text type, translation mode, and the nature of the translated target.

In this paper, this controversial issue is addressed by looking at the English translations of the Wolf Totem in both literary and subtitle situations. Specifically, we seek to understand how the culture-specific items that carry alien Chinese concepts are transferred into English. The dominant processing pattern is identified in both the literary and subtitle translation in sections 5.1 and 5.2, respectively; thus, the first hypothesis is confirmed. Moreover, based on the empirical evidence, it is found that the meaning-based processing route dominates both literary and subtitle translation; thus, the second hypothesis is rejected. It is further argued that the contextual-intentional-conceptual system handles culture-specific concepts through conceptual mediation, which is required in the translation process due to contextual and pragmatic factors.

To the best of our knowledge, this is the first study comparing subtitle translation with written translation in terms of the processing patterns. The issue of the cognitive processing routes that may take place in the translator's brain is of significance to translation process research specifically and translation studies in general.

\section{Funding}

This paper was supported by Scientific Research Fund of Hunan Provincial Education Department (Reference no: 19C0971).

\section{References}

[1] Aixelá, J. F. (1996). Culture-specific items in translation. In Alvarez, R. \& M. Vidal (Eds.), Translation, power, subversion (pp. 52-78). Beijing: Foreign Language Teaching and Research Press.

[2] Balling, L., Hvelplund, K., Sjrup, A. (2014) Evidence of Parallel Processing During Translation. Meta, 2, 234-259.

[3] Barik, H. C. (1975). Simultaneous interpretation: Qualitative and linguistic data. Language and Speech, 18 (3), 272-297. 
[4] Chou, I. C., Lei, V. L. C., Li, D., \& He, Y. (2016). Translational ethics from a cognitive perspective: A corpus-assisted study on multiple English-Chinese translations. In T. Seruya \& J. M. Justo (Eds.), Rereading schleiermacher: translation, cognition and culture (pp. 159-173). Berlin/Heidelberg: Springer.

[5] Chomsky, N. (2000). New Horizons in the Study of Language and Mind. Cambridge: CUP.

[6] Christoffels, I. K., \& de Groot, A. M. B. (2005). Simultaneous interpreting: A cognitive perspective. In J. F. Kroll \& A. M. B. De Groot (Eds.), Handbook of bilingualism: Psycholinguistic approaches (pp. 454-479). Oxford/New York: Oxford University Press.

[7] Dam, H. V. (1998). Lexical similarity vs lexical dissimilarity in consecutive interpreting: A product-oriented study of form-based vs meaning-based interpreting. The Translator, 4 (1), 49-68.

[8] Dam, H. V. (2001). On the option between form-based and meaning-based interpreting: The effect of source text difficulty on lexical target text form in simultaneous interpreting. The Interpreters' Newsletter, 11, 27-55.

[9] De Groot, A. M. B. (1997). The cognitive study of translation and interpretation: Three approaches. In J. H. Danks, G. M. Shreve, S. B. Fountain, \& M. K. McBeath (Eds.), Cognitive processes in translation and interpreting (pp. 25-56). Thousand Oaks/ London/New Delhi: Sage.

[10] De Groot, A. M. B. (2011). Language and cognition in bilinguals and multilinguals: An introduction. New York: Psychology Press.

[11] Dong, Y. \& Lin, J. (2013). Parallel processing of the target language during source language comprehension in interpreting. Bilingualism: Language and Cognition, 16, 682-692.

[12] García, A. M. (2015). Psycholinguistic explorations of lexical translation equivalents. Translation Spaces, 4 (1), 9-28.

[13] Ge, L. (2011). A corpus-based study on translating humorous texts: A case study on the English translation of the Chinese novel "Fortress Besieged" by Ch'ien Chung-shu (Unpublished doctoral dissertation). The Chinese University of Hong Kong, Hong Kong, China.

[14] He, Y. (2004). Mapping culturally indigenous concepts in the translation process: A cognitive perspective. Journal of Translation Studies, 9, 33-50.

[15] He, Y. (2009). Translating alien sources from and into Chinese: What does the translator do, and why? In X. Luo \& Y. He (Eds.), Translating China (pp. 207-232). Manchester: Multilingual Matters.

[16] He, Y. (2019). Translating and interpreting as bilingual processing: The theoretical framework. In Li, D., Victoria. Lei, $\&$ He. Y (eds.), Researching cognitive processes of translation (pp. 15-48). Singapore: Springer.

[17] Liu, X. (2018). How Do Interpreting Patterns Implicate Cognitive Processing Routes? Evidence from English vs Chinese Consecutive Interpreting, unpublished $\mathrm{PhD}$ Thesis, University of Macau, Macao, China.

[18] Liu, X., Li, D \& He, Y. (Forthcoming). Form-based or Meaning-based: A Corpus-assisted Study of Processing Routes in Consecutive Interpreting.

[19] Paradis, M. (1994). Toward a neurolinguistic theory of simultaneous translation: The framework. International Journal of Psycholinguistics, 10 (3), 319-335.

[20] Paradis, M. (2004). A neurolinguistic theory of bilingualism. Amsterdam/Philadelphia: John Benjamins.

[21] Pinker, Steven. (1999). Words and Rules: The Ingredients of Language. London: Pheonix.

[22] Tirkkonen-Condit, S. (2005). The monitor model revisited: Evidence from process research. Meta: Journal Des traducteurs Meta: Translators'Journal, 50 (2), 405-414.

[23] Zhou, J., \& He, Y. (2012). Naturalization as a translating strategy: On target cultural items in the source text. Translation Quarterly, 63, 50-67. 